\title{
Selective modulation of chemical and electrical synapses of Helix neuronal networks during in vitro development
}

\author{
Paolo Massobrio ${ }^{1 *+}$, Carlo NG Giachello ${ }^{2+}$, Mirella Ghirardi ${ }^{2,3}$ and Sergio Martinoia ${ }^{1}$
}

\begin{abstract}
Background: A large number of invertebrate models, including the snail Helix, emerged as particularly suitable tools for investigating the formation of synapses and the specificity of neuronal connectivity. Helix neurons can be individually identified and isolated in cell culture, showing well-conserved size, position, biophysical properties, synaptic connections, and physiological functions. Although we previously showed the potential usefulness of Helix polysynaptic circuits, a full characterization of synaptic connectivity and its dynamics during network development has not been performed.
\end{abstract}

Results: In this paper, we systematically investigated the in vitro formation of polysynaptic circuits, among Helix B2 and the serotonergic C1 neurons, from a morphological and functional point of view. Since these cells are generally silent in culture, networks were chemically stimulated with either high extracellular potassium concentrations or, alternatively, serotonin. Potassium induced a transient depolarization of all neurons. On the other hand, we found prolonged firing activity, selectively maintained following the first serotonin application. Statistical analysis revealed no significant changes in neuronal dynamics during network development. Moreover, we demonstrated that the cell-selective effect of serotonin was also responsible for short-lasting alterations in $\mathrm{C} 1$ excitability, without long-term rebounds.

Estimation of the functional connections by means of cross-correlation analysis revealed that networks under elevated $\mathrm{KCl}$ concentrations exhibited strongly correlated signals with short latencies (about $5 \mathrm{~ms}$ ), typical of electrically coupled cells. Conversely, neurons treated with serotonin were weakly connected with longer latencies (exceeding $20 \mathrm{~ms}$ ) between the interacting neurons. Finally, we clearly demonstrated that these two types of correlations (in terms of strength/latency) were effectively related to the presence of electrical or chemical connections, by comparing MicroElectrode Array (MEA) signal traces with intracellularly recorded cell pairs.

Conclusions: Networks treated with either potassium or serotonin were predominantly interconnected through electrical or chemical connections, respectively. Furthermore, B2 response and short-term increase in C1 excitability induced by serotonin is sufficient to trigger spontaneous activity with chemical connections, an important requisite for long-term maintenance of firing activity.

Keywords: Helix neurons, Micro-Electrode Arrays, Functional connectivity, Network development, Dynamics

\footnotetext{
*Correspondence: paolo.massobrio@unige.it

${ }^{\dagger}$ Equal contributors

${ }^{1}$ Neuroengineering and Bio-nano Technology Group (NBT), Department of Informatics, Bioengineering, Robotics, System Engineering (DIBRIS), University of Genova, Genova, Italy

Full list of author information is available at the end of the article
}

\section{() Biomed Central}

(c) 2013 Massobrio et al.; licensee BioMed Central Ltd. This is an Open Access article distributed under the terms of the Creative Commons Attribution License (http://creativecommons.org/licenses/by/2.0), which permits unrestricted use, distribution, and reproduction in any medium, provided the original work is properly cited. 


\section{Background}

In the past, the relative simplicity of the nervous system of invertebrates such as Helix snails allowed the detailed study of many aspects of neuronal connectivity [1-3], neurite outgrowth [4], synapse formation and plasticity [4-7] by using an in vitro approach (reviewed in [8]). In this experimental preparation, a large number of neurons can be individually identified and isolated in cell culture. The study of reciprocal interactions among identified neurons in small reconstructed circuits was pioneered in 1990 by Kleinfeld et al. [9] by using Aplysia neurons. More recently, the connectivity between $\mathrm{C} 1$, C3, and B2 Helix neurons was first investigated at the level of micro-networks (i.e., pairs of synaptically connected neurons, whose activity is recorded by means of intracellular sharp electrode techniques) [5], and later at the level of large-networks [10], by exploiting the technology offered by Micro-Electrode Arrays (MEAs). In vivo, the serotonergic $\mathrm{C} 1$ neurons, localized in the ventral side of cerebral ganglia, are synaptically connected to B2 neurons in the buccal ganglia [11], and they are involved in the regulation of feeding behaviors of Helix snails. In this way, it is feasible to reconstruct in dissociated cell culture synaptic connections among individually identified invertebrate neurons that resemble in vitro their in vivo features [4]. Helix neurons are large in size (soma diameter up to 100-150 $\mu \mathrm{m}$ ) compared with the microelectrode diameter $(30 \mu \mathrm{m})$ of MEAs. This geometrical ratio makes possible a 1:1 coupling between neurons and microelectrodes (not easy to do with mammalian neurons), facilitating the study of the relationship between the electrophysiological activity of individual neurons in a network, as well as the development of neurite outgrowth. However, the aforementioned studies were acutely performed, recording the electrophysiological activity for a few hours once the circuit has been established.

To the best of our knowledge, in vitro studies about long lasting (i.e., days) recordings of invertebrate neuronal networks cannot be found. The possibility to couple Helix neurons to MEAs, allows recordings of the electrophysiological activity and monitoring the development of network organization can modulate such activity in the long term.

Indeed, MEA technology is the "gold standard" to study the development of in vitro neuronal networks, as demonstrated by many studies related to mammalian cortical networks [12-16]: during development, while synapses and neural connectivity build up, neuronal network activity starts to self-organize [17], and modulates its electrophysiological patterns (i.e., spike and bursts).

Following this approach, the purpose of this work is to study and characterize the dynamics and connectivity of
Helix networks made up of C1 and B2 neurons coupled to MEAs during their development.

Helix neurons start to create synaptic connections just a few hours after plating, and their development is faster (couple of days) than cortical cultures from mammals (3-4 weeks in vitro) [13]. A peculiarity of these invertebrate neuronal networks is the absence of spontaneous activity: the Helix neurons used in this study were found to be generally silent on MEAs $[10,18]$, and spontaneous firing was observed only occasionally. Therefore, we triggered neuronal activity by means of chemical treatments, namely potassium chloride $(\mathrm{KCl})$ which induces general depolarization of the cell membrane potential and serotonin (5-HT) which selectively depolarizes B2 neurons. Thus, the induced activity was followed during development at fixed time-points (i.e., 6, 24, 30, 48, 54, 72 hours after cell plating) and analyzed by means of the firstorder statistics used to characterize neuronal dynamics: inter spike interval (ISI) and firing rate. The estimation of the functional connections established among neurons of the network was made by using cross-correlation function [19] in order to reconstruct the topological connections, and monitor them during development. Considering the simplicity of these neuronal circuits, a comparison with the morphological development of the network was possible, and a good matching between morphological and functional links was achieved.

We found that both chemical stimulations (i.e., $\mathrm{KCl}$ and 5-HT) were efficacious to trigger firing activity in Helix circuits, but a long-lasting change in activity occurred only with 5-HT treatment. We did not observe a modulation of the dynamics as far as the network evolved, although we counted an increase with time of functional connections. Moreover, the analysis of spiking activity as well as functional link latencies suggest that networks treated with 5-HT displayed a dynamic modulated mostly by chemical synapses, while a predominance of electrical connections occurred in $\mathrm{KCl}$-triggered networks.

\section{Results}

Experimental data collected by MEAs and presented in this work were obtained from 8 Helix cultures, monitored at the $6,24,36,48,54$, and 72 hours after plating. As explained in the Methods Section, we considered 3 cultures treated with serotonin (5-HT), 2 with extracellular high-potassium $(\mathrm{KCl})$, and 3 as control, i.e., without any kind of treatment.

Helix polysynaptic circuits display a treatment-dependent long-lasting activity

When Helix neurons are cultured in vitro, they do not display any kind of electrical spontaneous activity [10]. We followed the development (from 6 to 72 hours after 
plating) of 3 cultures made up of $12 \mathrm{~B} 2$ and $4 \mathrm{C} 1$ neurons arranged in the configuration depicted in Figure 1A. At each time-point, we recorded $30 \mathrm{~min}$ of spontaneous activity: no spike activity was observed in the 3 non stimulated cultures. To verify the healthy conditions of the networks, and the effective presence of synaptic connections, we delivered electrical stimulation at the $72^{\text {nd }}$ hour. Selected cells were singularly impaled and stimulated with a current pulse whose amplitude was sufficient to overcome the excitability threshold. Under these conditions, we were able to observe a network activity which involved all neurons of the network (data not shown).

To trigger electrophysiological activity in Helix networks, we chemically treated such cultures by means of two stimulation protocols at each time-point of recording: i) Increasing extracellular potassium concentration (KCl) from $5 \mathrm{mM}$ to $65 \mathrm{mM}$, that results in a general depolarization of the membrane potentials of cells; ii) delivery of serotonin (5-HT) in a concentration equals to $20 \mu \mathrm{M}$ that selectively depolarizes B2 neurons. The details of these protocols are described in the Methods Section.

Figure 2 shows the qualitative results from one culture treated with $\mathrm{KCl}$. Each horizontal block of the figure represents a recording time-point (i.e., 6, 24, 36, 48, 54, 72 hours). The three raster plots per each block show one minute of electrophysiological activity before, during and after the $\mathrm{KCl}$ delivery. Red and blue lines are for $\mathrm{C} 1$ and B2 neurons, respectively. The first observation is that $\mathrm{KCl}$ induced a transient depolarization of the network which lasted no more than 1 minute: neurons quickly depolarized with a delay of $825 \pm 43 \mathrm{~ms}$ from the $\mathrm{KCl}$ delivery. As a result of the sustained membrane depolarization, networks moved toward a quiescent state before the wash-out, possibly through the inactivation of voltage-gated sodium channels. The phases before and after the $\mathrm{KCl}$ stimulation were mainly characterized by the absence of activity: before the stimulation, Helix networks were always completely silent (cf., also the IFR graph reported in Figure $3 \mathrm{~A}$ ). After the wash-out the network reached a resting state, where no activity was recorded: only little oscillations in the B2 neurons were occasionally found, as shown in Figure 3A. The main observation from these raster plots and from the IFR curves from 24-72 hours of development is that $\mathrm{KCl}$ does not induce persistent activity in the network. This transient behavior was constant for all the periods of recording during development.

Conversely, a long-lasting neural activity in networks treated with 5-HT were observed. Figure 4, arranged with the same graphical layout of Figure 2, shows an example of Helix network treated with 5-HT. Except for the raster plots of the first block (first and third column, i.e., before and after the 5-HT stimulation, respectively), neurons continued to fire after the wash-out (third column), and several hours later (first column). The delivery of 5-HT seems to trigger the neuronal network circuits towards an oscillatory state which persists for long-term during network development. We estimated the frequency of the quasi-periodic behavior of the $\mathrm{C} 1$ and B2 cells by computing the reciprocal of the ISI. Once entered into a periodic regime, we found that $\mathrm{C} 1$ neurons fired at a frequency which range from $0.2 \mathrm{~Hz}$ during the pre-stimulation phase, to $0.6 \mathrm{~Hz}$ during the stimulation and then decreasing to $0.3 \mathrm{~Hz}$ in the post stimulus phase. These values were maintained during the network development. B2 neurons exhibited a firing frequency of about $1 \mathrm{~Hz}$ during the periodic regime. To assess whether the firing activity was periodic, we computed the coefficient of variation $(\mathrm{CV})$ of the ISI. By setting an arbitrary threshold at 0.2 , we considered quasi-periodic those neurons which presented a CV less than 0.2. Additional file 1 shows the trends of both the $\mathrm{CV}$ and the frequency. The networks treated with $\mathrm{KCl}$ never displayed a quasi-periodic behavior, since CV was always greater than 0.2 .
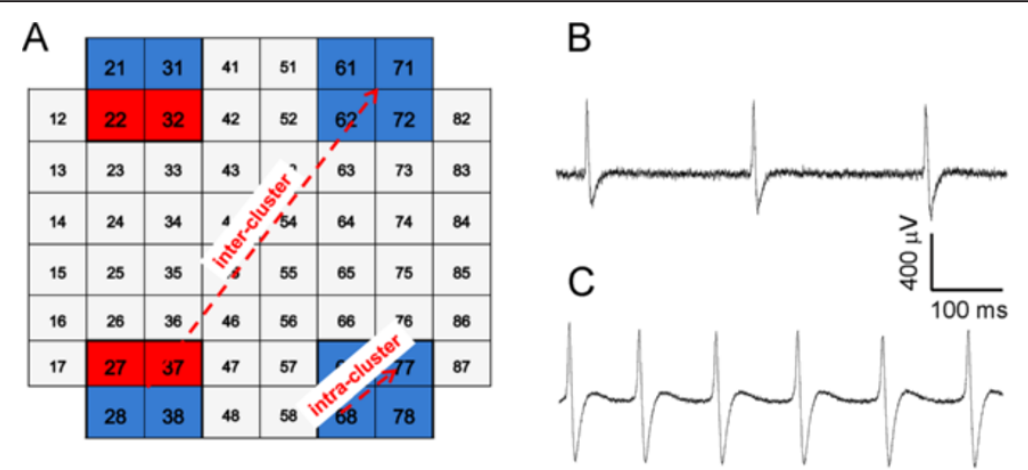

Figure 1 Schematic representation of the network organization and raw data. (A) Sample scheme of neuron placement onto a MEA. Red and blue squares indicate the position of $\mathrm{C} 1$ and B2 cells, respectively. The number inside the squares indicate the electrode position in the MEA layout. (B) 1 second of activity from a microelectrode with a C1 neuron. (C) 1 second of activity coming from a microelectrode with a B2 neuron. 


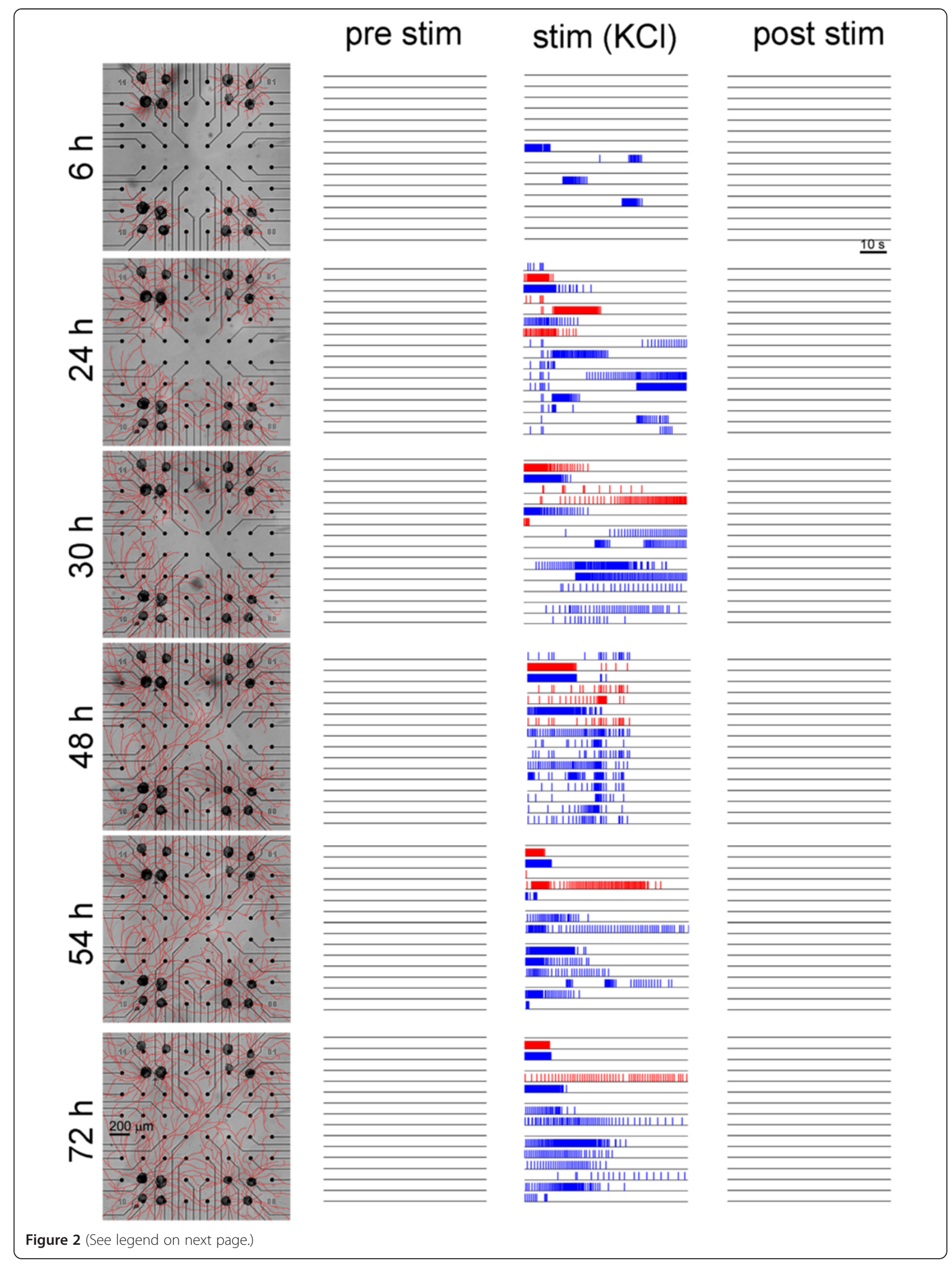


Figure 2 Example of Helix culture manipulated with $\mathrm{KCl}$ applications. Each block represents a time-point of recordings (i.e., 6, 24, 36, 48, 54, 72 hours after plating). In the first column, the development of the neurite arborizations is indicated (scale bar is $200 \mu m$ ). The three columns of raster plots show one minute of electrophysiological activity just before, during and after the $\mathrm{KCl}$ treatment. Red and blue lines indicate $\mathrm{C} 1$ and B2 neurons respectively. Scale bar is $10 \mathrm{~s}$.

To better analyze the mechanisms underlying 5-HTinduced long-lasting activity, we further characterized Helix networks in order to evaluate whether this event is related to changes in cell excitability or, alternatively, to modifications of morphological and functional connectivity.

\section{Helix networks show stable dynamics during development}

Following the approach used to characterize the in vitro development of mammalian cortical neurons coupled to MEAs [12,13], we first quantified the mean firing rate (MFR) and the inter spike interval (ISI). Figure 3A and B shows the IFR trends of the $\mathrm{C} 1$ (red) and B2 (blue) neurons. By comparing the IFR trends of the cultures treated with $\mathrm{KCl}$ (Figure 3A) and 5-HT (Figure 3B), the main difference was the total absence of electrophysiological activity in both $\mathrm{C} 1$ and $\mathrm{B} 2$ neurons before the $\mathrm{KCl}$ stimulation. On the contrary 5 - HT induced a lowfrequency $(<5 \mathrm{~Hz})$ baseline activity, especially in the B2 cells. During development, no remarkable differences were noticed: in cultures stimulated with $\mathrm{KCl}$, we observed a rapid increase of activity immediately after the stimulation, which lasted less than 1 minute. Both in $\mathrm{C} 1$ and $\mathrm{B} 2$ neurons, the IFR reached a maximum value of $20 \mathrm{~Hz}$. In the case of 5-HT stimulation the IFR after a sudden increase remained stable through all the stimulation phase. No main differences were observed during development. $\mathrm{C} 1$ and $\mathrm{B} 2$ neurons fired in a very regular way: B2 neurons fired at a higher frequency than $\mathrm{C} 1$, and this activity remained until the wash-out. Afterwards, after a brief transient (from 2 to 5 minutes)

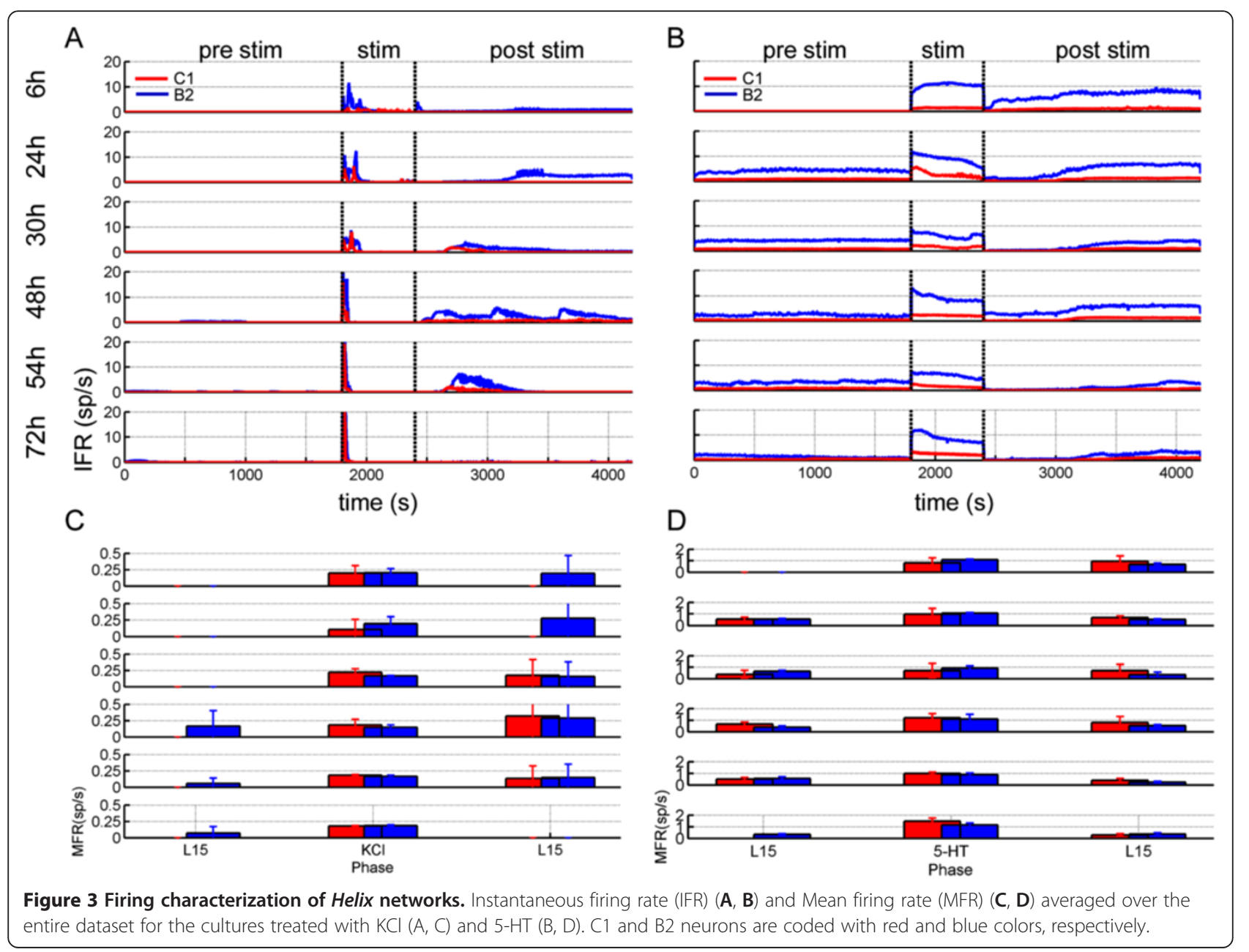




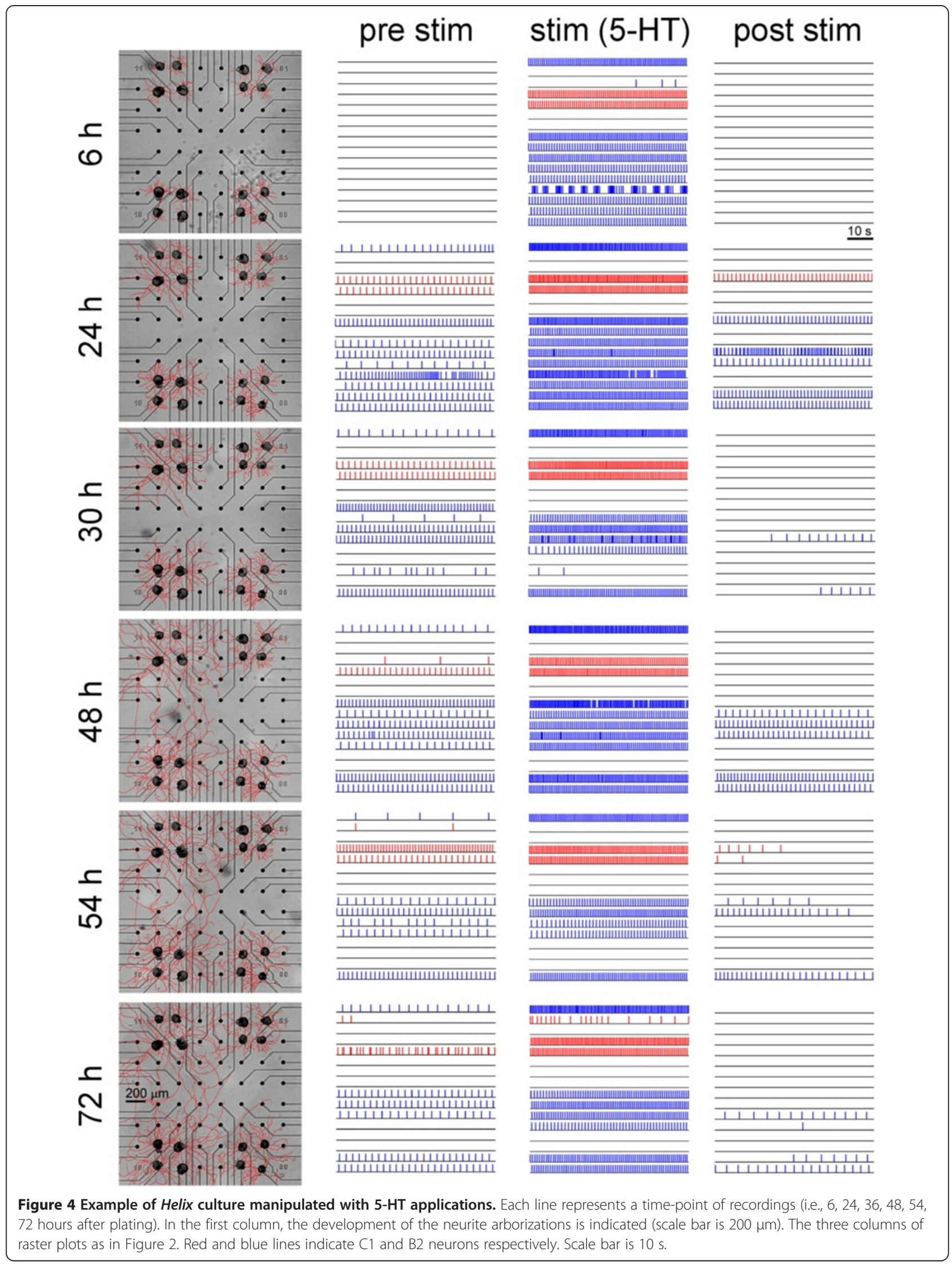


characterized by a silent period, B2 neurons spontaneously restarted to fire. In this post stimulus phase, the dynamics exhibited by the networks treated with $\mathrm{KCl}$ was more irregular: $\mathrm{C} 1$ neurons were always quiescent during development, whereas B2 ones showed a peak of activity at the $48^{\text {th }}$ hour. Then, the IFR slowed down at $54^{\text {th }}$ hour to zero at the $72^{\text {nd }}$ hour.

By quantifying the amount of spikes generated by networks stimulated by $\mathrm{KCl}$ or 5-HT during development, we computed the MFR as depicted in Figures $3 \mathrm{C}$ and $3 \mathrm{D}$. Averaged over a time window of 30 minutes, the MFR peaks for the Helix neuronal networks treated with $\mathrm{KCl}$, and $5-\mathrm{HT}$ reached values of 0.4 and $1.5 \mathrm{sp} / \mathrm{s}$, respectively. In particular, we found a maximum value of the MFR for the network treated with 5-HT equals to $1.16 \pm 0.16$ (B2 neurons) and $1.48 \pm 0.27$ ( $\mathrm{C} 1$ neurons) $\mathrm{sp} / \mathrm{s}$ during the stimulation phase at the $72^{\text {nd }}$ hour timepoint. For the $\mathrm{KCl}$ manipulation, a peak in the firing activity of $0.29 \pm 0.41$ (B2 neurons) and $0.32 \pm 0.45$ (C1 neurons) $\mathrm{sp} / \mathrm{s}$ was reached after 48 hours from the plating in the post stimulation phase. Interestingly, MFR values were statistically equal during development (Figures $3 \mathrm{C}$ and $\mathrm{D}$ ), suggesting that spiking activity was stable although the network connectivity changed and evolved (cf., Figures 2, 4, and 7).

To better quantify possible changes in the network dynamics during in vitro development, we derived the inter spike interval (ISI) distribution as shown in Figure 5. Figures $5 \mathrm{~A}$ and $\mathrm{B}$ show the ISI distributions relative to the stimulation phases (i.e., 5- $\mathrm{HT}$ (gray), $\mathrm{KCl}$ (black)) for $\mathrm{C} 1$ and $\mathrm{B} 2$ neurons, respectively. We found that $\mathrm{KCl}$ induced a faster spiking activity than 5-HT: both $\mathrm{C} 1$ (Figure 5A) and B2 (Figure 5B) neurons when stimulated with $\mathrm{KCl}$ (black line) presented a main ISI peak less than $0.5 \mathrm{~s}$, while the 5 -HT treatment (gray line) shifted such peaks for both $\mathrm{C} 1$ and $\mathrm{B} 2$ neurons towards $1 \mathrm{~s}$. The latency of the main peak (preferred inter-spike interval) showed no statistically significant differences during development both for $\mathrm{C} 1$ and B2 neurons and for both stimulations (cf. Figure 5C and D). Thus, comparing the
A

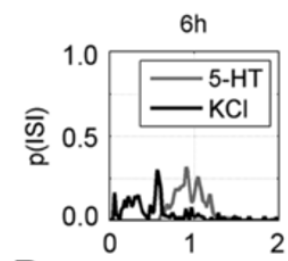

B
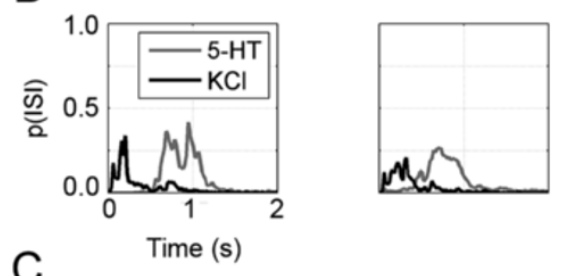

C1 neurons

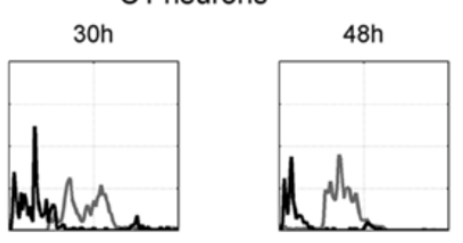

B2 neurons
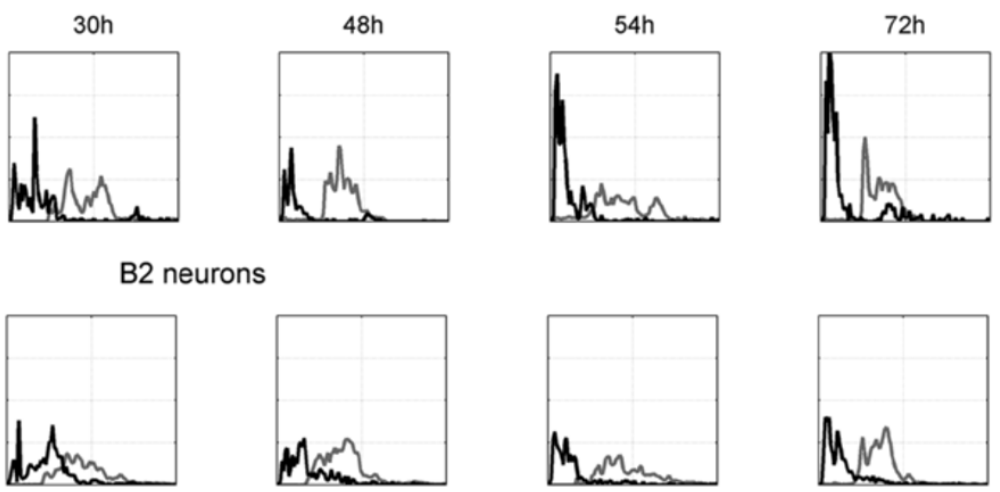

C

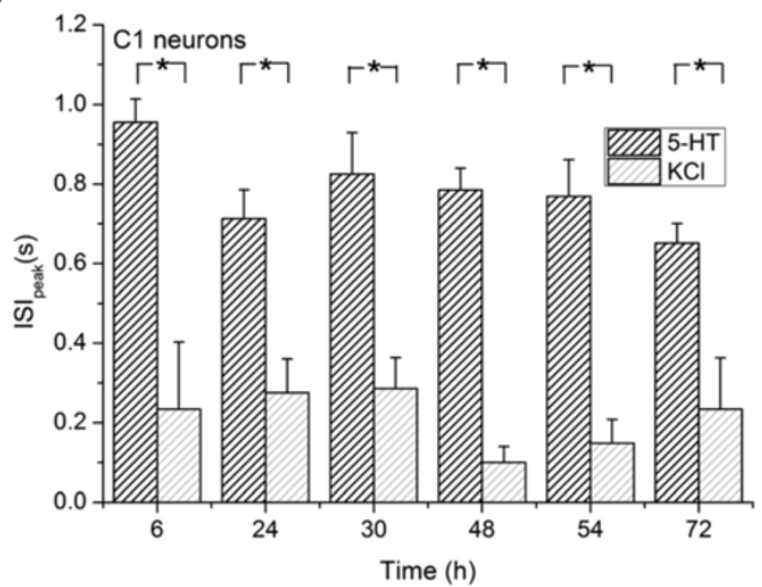

D

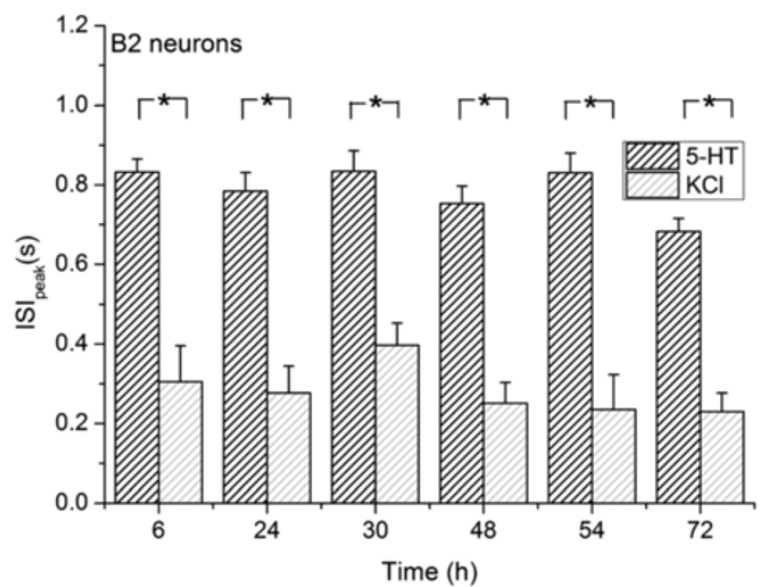

Figure 5 Inter spike interval (ISI) characterization of Helix networks. (A, B) ISI distribution relative to the stimulation phases for (A) C1 and (B) B2 neurons. The treatment with $5-\mathrm{HT}$ and $\mathrm{KCl}$ is coded with gray and black colors, respectively. Bin width = $20 \mathrm{~ms}$. (C, D) Temporal peak position of the ISI distributions relative to C1 (C) and B2 (D) neurons, respectively. (Kruskal-Wallis, non-parametric test, P < 0.01 ) 
cultures treated with $\mathrm{KCl}$ and 5-HT, we found a statistically significant difference in the ISI temporal peak position (Kruskal-Wallis, non-parametric test, $P<0.01$ ).

\section{Synaptically isolated Helix neurons do not show excitability changes with $5-\mathrm{HT}$ and $\mathrm{KCl}$ treatments}

In order to evaluate 5 - $\mathrm{HT}$ or $\mathrm{KCl}$-induced alterations in neuronal excitability, we measured biophysical parameters on cultured isolated $\mathrm{C} 1$ and $\mathrm{B} 2$ neurons, by using intracellular recording techniques. The same cell culture procedures and treatments described for MEA devices were applied to these cells. We analyzed both spontaneous and firing activity induced by a depolarization stimulus applied at 6 hours after plating, before the treatment and soon after the treatment washout (Figure 6A). Moreover, the same cells were recorded again at 24 hours after plating to check the presence of long-term effects. Generally, at 6 hours after plating, B2 neurons displayed a low-frequency spontaneous activity before the treatment $(n=8$ in each group; Figure 6B). These cells strongly increased their firing activity after the washout of $20 \mu \mathrm{M}$ serotonin applied for 10 minutes, switching their firing rates from $0.06 \pm 0.06$ spikes $/ \mathrm{s}$ to $0.72 \pm 0.14$ spikes $/ \mathrm{s}$ (vs. $0.12 \pm 0.07$ spikes/s in control group; $P<0.001$, Bonferroni's post-hoc test). No significant change was observed after the washout of high- $\mathrm{KCl}$ solution, thus confirming that washout completely depleted the potassium-induced activity. The same cells were tested again at 24-hour time-point and no spontaneous activity was detected in all the experimental groups. On the other hand, C1 neurons were always completely silent (Figure 6C).

These observations indicate that Helix isolated cells did not show any prolonged spontaneous activity triggered by 5 -HT application, differently from polysynaptic circuits recorded on MEA devices (Figure 4).

In addition, we also investigated whether the activity of 5-HT-treated circuits may be ascribed to changes in cellular excitability by considering the firing rate of single cells induced by a depolarization pulse $(+0.5 \mathrm{nA}$ for $10 \mathrm{~s})$. Isolated B2 neurons showed the same response in all experimental groups (Figure 6D). Interestingly, we observed a general increase in excitability from 6- to 24-hour timepoints in $\mathrm{C} 1$ neurons (Figure 6E). A two-way ANOVA for repeated measures confirmed a significant effect of time $\left(\mathrm{F}_{(2,25)}=34.39, P<0.0001\right)$. Here, the application of serotonin induced a rapid and strong increase in firing rate that is statistically significant if compared to control and KCl-treated groups (5-HT: $3.300 \pm 0.661$ spikes/s, $n=11$; vs. control: $0.357 \pm 0.192$ spikes/s, $n=7$; vs. $\mathrm{KCl}$ : $0.110 \pm$ 0.023 spikes/s, $n=10 ; P<0.001$, Bonferroni's post-hoc test). Thus, we can infer that $\mathrm{C} 1$ neurons bear receptors responsive for serotonin that are involved in regulating their excitability without modifying their membrane potential, as observed in the homologous Aplysia metacerebral cell $[20,21]$.

In all experimental groups, we observed no changes in other membrane properties, such as input resistance, threshold potential and resting potential (data not shown). We further evaluated the presence of alterations in action potential (AP) kinetics, measuring peak amplitude, AP half-width duration, AP rise time, AP decay time, after-hyperpolarization and rheobase. For each parameter, no difference was found between treated and control groups (data not shown).

\section{5-HT and $\mathrm{KCl}$ treatments modulate neurite outgrowth in Helix circuitry}

To better understand firing dynamics and functional connections inferred from MEA recordings, a parallel evaluation of neurite outgrowth and morphological network was performed. We measured the number of neurites interconnecting neurons within the same cluster (intra-cluster) and among the 4 different clusters the neurite density (inter-cluster) to evaluate neurite densities. Originally, data measured from $\mathrm{C} 1$ and B2 neuron have been analyzed separately: we decided to include these values in the same group since their growth rates were very similar to each other.

As shown in Figure 7A, we found a general and rapid increase of intra-cluster neurite density with time reaching a limiting plateau value determined by the restricted surface area encompassed by each cluster (distance between two electrodes $=200 \mu \mathrm{m})$. This parameter is treatmentdependent. A two-way ANOVA for repeated measures revealed a significant effect of the treatment $\left(\mathrm{F}_{(2,29)}=\right.$ 48.53, $P<0.0001)$ and time $\left(\mathrm{F}_{(2,6)}=466.7, P<0.0001\right)$ and a significant treatment by time interaction $\left(\mathrm{F}_{(12,174)}=\right.$ 17.12, $P<0.0001)$. Particularly, we observed a statistically significant decrease of intra-cluster neurite density in 5-HT-treated circuits $(n=12)$ that started from 24 hours and persisted at the following time-points reaching the value of $0.029 \pm 0.002$ neurites $/ \mu \mathrm{m}^{2}$ vs. $0.040 \pm 0.002$ neurites $/ \mu \mathrm{m}^{2}$ of control group $(n=12)$ at 72 hours $(P<$ 0.001 , Bonferroni's post-hoc test). Conversely, the application of high-potassium solution induced an increase in neurite density with $0.051 \pm 0.001$ neurites $/ \mu \mathrm{m}^{2}$ measured at 72 hours ( $n=8 ; P<0.001$, Bonferroni's post-hoc test).

We also considered the inter-cluster neurite density as an index of long-range neurites reflecting the putative connections among the four clusters (Figure 7B). During the first hours of development, none or few interconnecting neurites were observed confirming that signal propagation in the corresponding recordings was restricted to the cluster itself. Inter-cluster neurite density linearly increased from 24 to 72 hours with similar values in the $\mathrm{KCl}$-treated and the control groups. The distance between clusters (600-1000 $\mu \mathrm{m})$ 


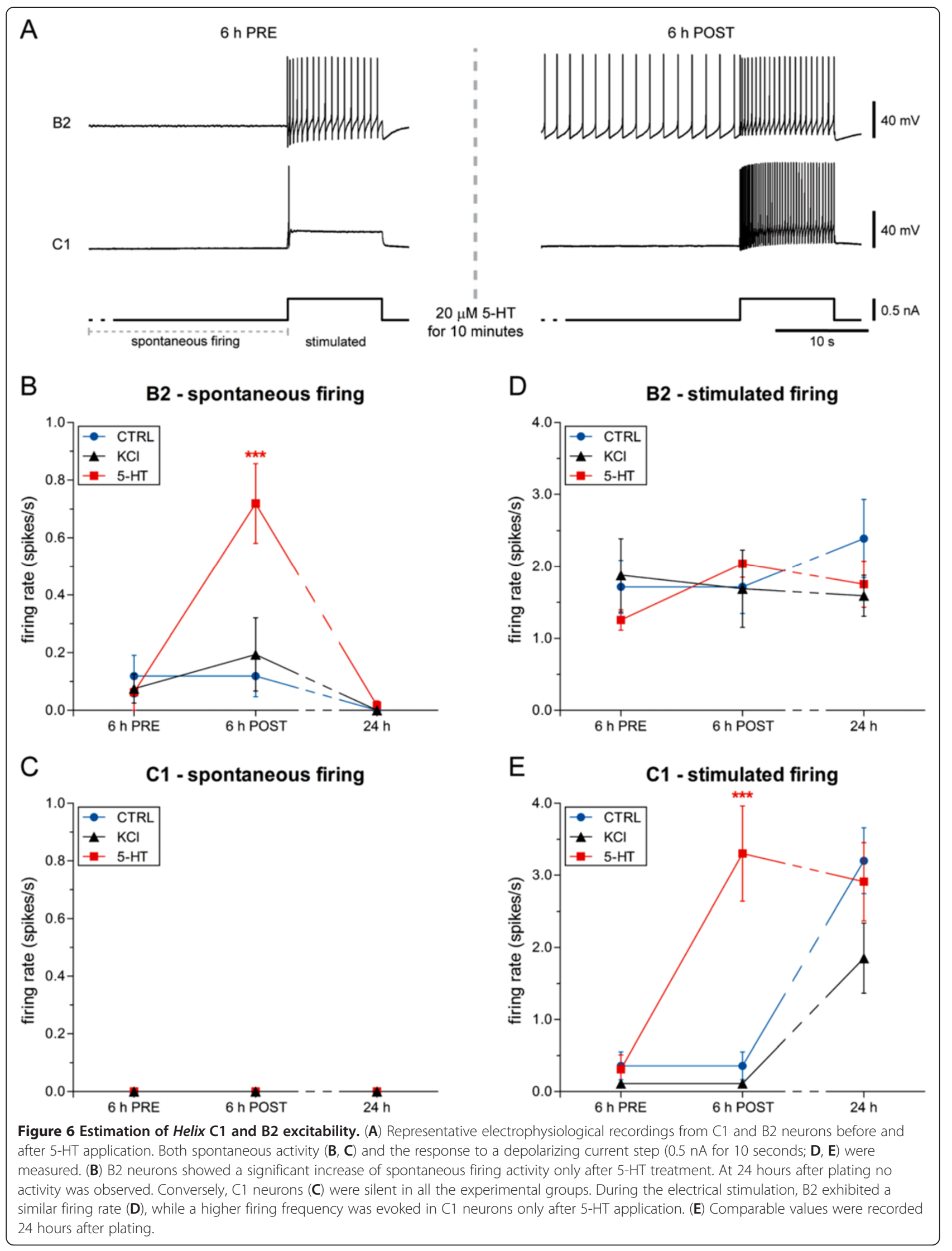




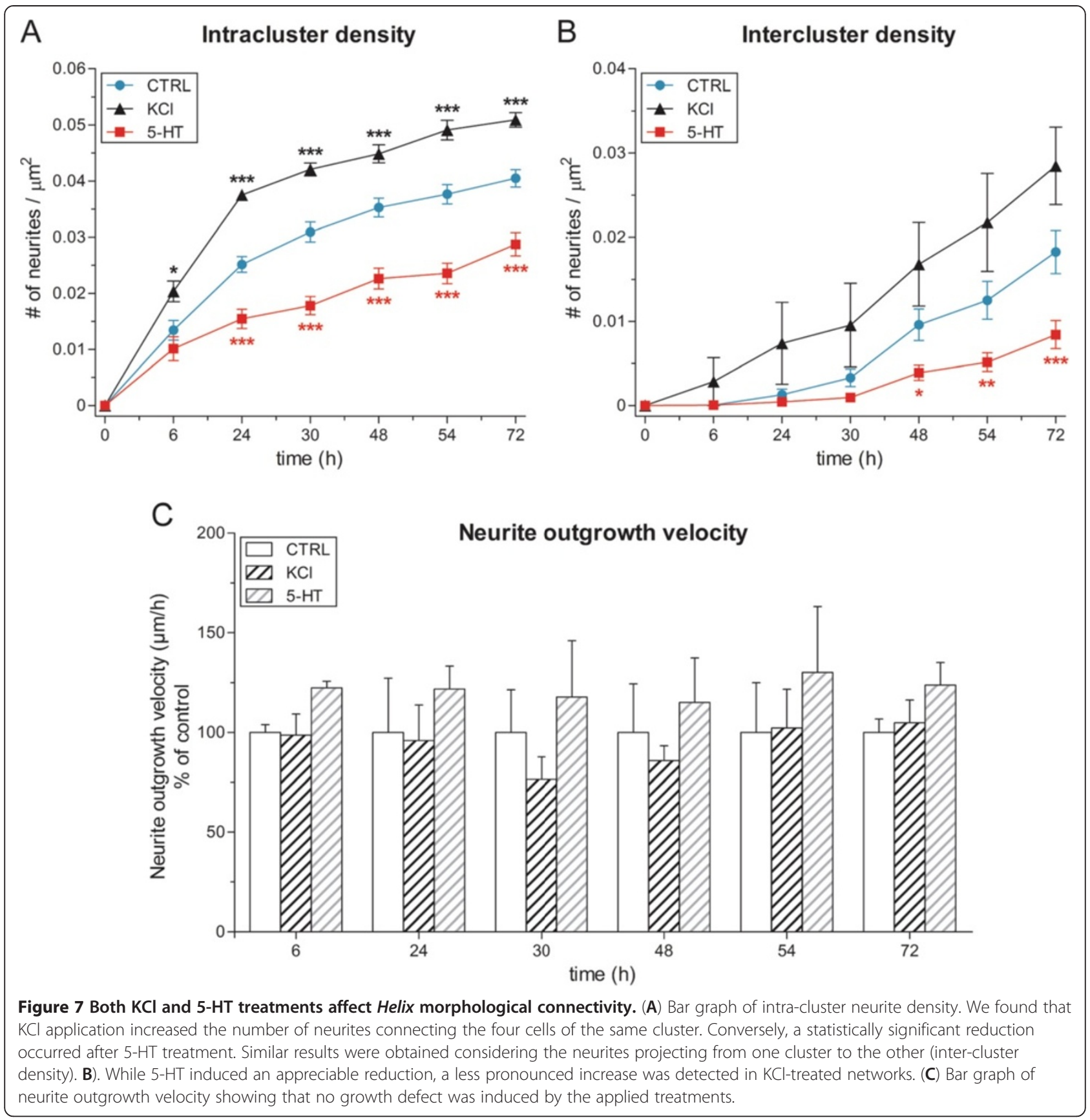

guaranteed a continuous neurite extension without imposing a plateau value. We found that this enhancement was significantly impaired by the application of serotonin (i.e. at 72 hours: $0.008 \pm 0.002$ neurites $/ \mu \mathrm{m}^{2}$ vs. $0.018 \pm 0.003$ neurites/ $\mu \mathrm{m}^{2}$ of control group; $P<0.001$, Bonferroni's post-hoc test).

To directly assess the presence of a growth deficit induced by a single serotonin application, we measured the velocity of neurite elongation at the different time-points. Similar values were found in all experimental groups suggesting that the motility of growth cone has not been affected (Figure 7C). Thus, the lower neurite density may be ascribed to a direct effect of serotonin rather than a growth impairment, as observed in other models [22-24].

\section{Helix networks display functional connections during development}

To investigate the overall circuit connectivity, we analyzed whether the synaptic connections detected in complex polysynaptic circuits of $\mathrm{C} 1$ and $\mathrm{B} 2$ neurons were formed according to previous electrophysiological characterizations of individual monosynaptic pairs [5] and whether the different treatments (i.e., $\mathrm{KCl}$ or 5-HT) 
modulate the functional connections, considering the evidence that neurite density is facilitated with $\mathrm{KCl}$ and depressed with 5-HT (cf., Figures 7A and 7B).

Figures 8 show two examples of functional connectivity (FC) maps relative to $\mathrm{KCl}$ (Figure 8A) and 5-HT (Figure 8B) treatments evaluated at the $48^{\text {th }}$ hour after plating. Two representative Cross-Correlations (CCs) were reported near the correspondent functional links: a relevant difference between $\mathrm{KCl}$ and 5-HT treatment on the cross-correlograms is the peak amplitude (about one order of magnitude; cf., also Figure 9 for a detailed characterization). FC maps were inferred from the CCs evaluated among all the active electrodes. Following the approach proposed in [25,26], we sorted all the statistically significant links based on the connection strengths, and we took into account only the 8 strongest links. In this way, it is likely we are considering the most reliable connections that would correspond to physical connections. From the FC analysis we did not find any relevant inter-cluster functional links even if we observed some long range connections in the morphological analysis (Figure 7B). The interaction measured by means of $\mathrm{CC}$ suggests that such distant neurons are not functionally connected. In fact, we found among inter-cluster electrodes: i) noisy and not significant CCs; ii) CC peaks falling into the bin centered in zero, thus representative of a random co-activation [26].

\section{5-HT and $\mathrm{KCl}$ treatments modulate the synaptic strength and latency}

As anticipated in the previous section, there is evidence that the two treatments modulate the FC of Helix neuronal networks. Thus, to characterize the functional links we estimated, for all the cultures and during their development, the strength (i.e., cross-correlogram peak value), and latency (i.e., the time difference between the occurrence of the cross-correlogram peak and zero).

Figure 9A shows the number of detected connections as a function of the network development, averaged over the number of cultures (i.e., 3 and 2 networks per 5-HT and $\mathrm{KCl}$ chemical treatment, respectively). All the detected connections were intra-cluster, since no intercluster links were found. Practically, this means that we were dealing with 12 and 8 not-functionally connected networks treated with $5-\mathrm{HT}$ and $\mathrm{KCl}$, respectively. $\mathrm{KCl}$ induces an increasing of the number of links compared with the networks manipulated with 5-HT showing similar results to that from measurement of neurite density (Figure 6A). After 54 hours in vitro, the networks treated with $\mathrm{KCl}$ reached a steady-state value of functional connections, while a linear monotonic trend was observed in the case of 5-HT. Interestingly, $\mathrm{KCl}$-mediated connections were stronger than the 5 -HT ones (after 6 hours in vitro). The trend of the estimated synaptic strength showed a peak at around the $30^{\text {th }}$ hours $(0.56 \pm 0.09)$, and converged towards an asymptotic value $(0.30 \pm 0.003)$. On the contrary, the estimated synaptic strength of the 5-HT networks was substantially flat with little oscillations and a small value around 0.03 (one order of magnitude smaller than $\mathrm{KCl}$ stimulation).

Next, we characterized the functional connections as latencies between the cross-correlated electrodes. $\mathrm{KCl}$-treated networks presented a relatively fast synaptic transmission (about $5 \mathrm{~ms}$ ) which remained rather constant during the development (Figure 9C). In the 5-HT treatment, the synaptic transmission was much slower: after 6 hours in vitro, the average latency was already at a plateau value of $20.4 \pm$ $0.43 \mathrm{~ms}$.

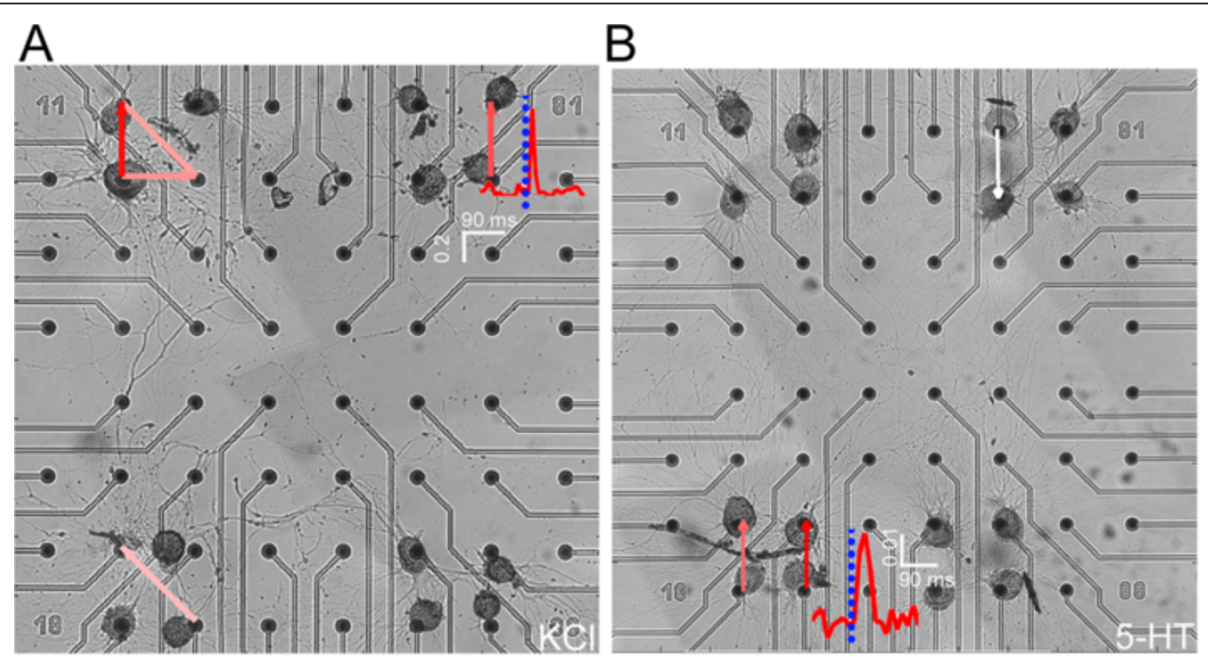

Figure 8 Functional connectivity maps. Examples of functional connectivity maps evaluated for the strongest 8 connections in a culture manipulated with (A) $\mathrm{KCl}$ or (B) 5-HT. The connection strength is coded by the color arrow: from red (strong) to white (weak). Inside the maps, two examples of cross-correlograms are reported. Scale bars: A: $x$-axis: 90 ms, $y$-axis: 0.2. B: $x$-axis: 90 ms, y-axis: 0.01. 


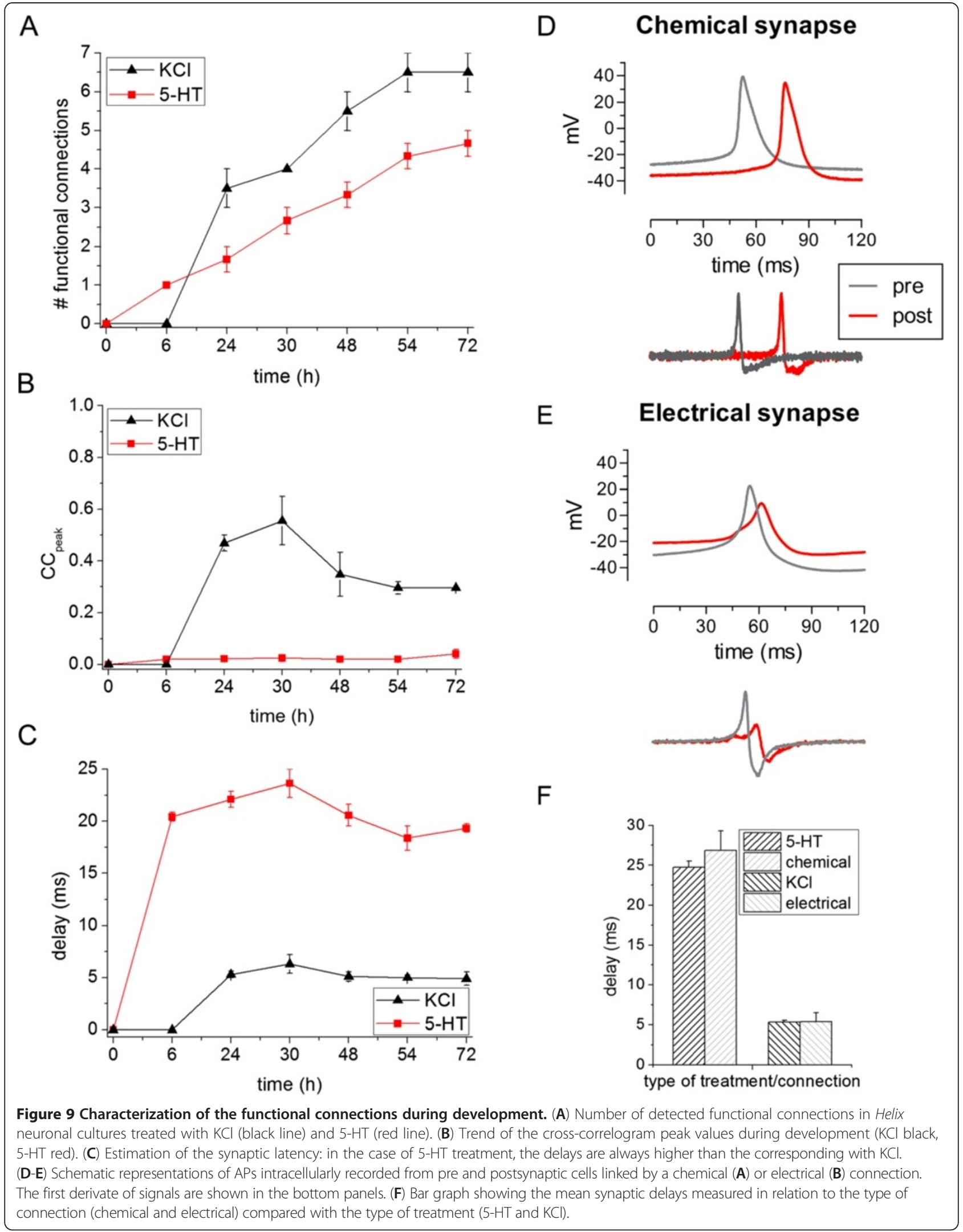


From the literature, it is known that Helix neurons may form both chemical and electrical synapses in culture [5]. Therefore, we consider the possibility that the two ranges of latencies measured may be related to the type of connection, more particularly to the synaptic delay. To sustain this hypothesis, we tested isolated C1-B2 and B2-B2 neuronal pairs for the presence of synaptic connections. Intracellular recording data of firing neurons were computationally transformed into the first derivative to reproduce extracellular signals acquired by MEA devices (Figure 9D and E). This manipulation is justified by previous modeling studies in which the neuron-microtransducer junction and the extracellular recorded waveform were mathematically modeled and characterized [27-29]. Then, we measured the delay time which occurred between two APs, one in the presynaptic cell and the other in the postsynaptic neuron triggered by the first signal (see Methods). We observed that cells electrically interconnected always fired in a synchronous fashion with a 1:1 relationship. Conversely, the action potentials recorded from chemically interconnected neurons were not always related to each other. In fact, an excitatory postsynaptic potential (EPSP) elicited by presynaptic firing may not be sufficient to trigger a new action potential in the postsynaptic neuron. For instance, EPSPs may not reach the threshold potential or, alternatively, may occur during the repolarization phase, when neuron is refractory to stimulation. These signals would result in membrane potential fluctuations that cannot be detected on MEA devices. Therefore, we decided to include in this analysis only spikes that are effectively correlated to each other. We found that chemical and electrical synapses are characterized by delays equal to $26.9 \pm 2.5$ $\mathrm{ms}$ and $5.4 \pm 1.1 \mathrm{~ms}$, respectively (Figure 9F). Such values are in agreement with the ones found at the network level (24.7 $\pm 0.8 \mathrm{~ms}$ and $5.3 \pm 0.3 \mathrm{~ms}$ average values after 48 hours) when treated with $5-\mathrm{HT}$ and $\mathrm{KCl}$, respectively.

Taken together, these data suggested that $\mathrm{KCl}$-treated Helix networks were prevalently interconnected through electrical synapses characterized by robust signals, synchronous firing and a strong coupling with short latency. On the other hand, polysynaptic circuits in which 5-HT has been repeatedly applied were formed by chemical connections, explaining a weaker connectivity and the longer latency. Therefore, we can infer that the enhancement of electrical versus chemical transmission in Helix circuits may be strongly up-regulated by means of $\mathrm{KCl}$ adding, and vice versa in the case of $5-\mathrm{HT}$.

\section{Discussion}

In this work, we studied the electrophysiological activity and dynamics of neuronal ensembles made up of $\mathrm{C} 1$ and B2 Helix neurons during their in vitro development. The advantage of this biological preparation is the predetermined knowledge of cell type composition, biophysical properties and connectivity. In addition, by exploiting the MEA technology (i.e., extracellular recordings), we were able to perform long-lasting measures in order to characterize the development of Helix networks.

Here, the absence of spontaneous activity [10,30] was observed even during the formation of synaptically interconnected clusters, despite the increasing neuritic growth and arborization, and the establishment of synaptic connections. Taking into account this feature, we decided to trigger electrophysiological activity by applying two chemical treatments: $\mathrm{KCl}$, to promote a general depolarization (unspecific stimulation); and 5-HT, to activate serotonergic receptors which are selectively expressed on B2 neurons. Following these stimulations, we observed similar dynamics during the development of these invertebrate circuits: the network firing early reached a value of firing rate and ISI, which remained almost unchanged during the development despite the connectivity maturation. This behavior is very peculiar, especially if compared with studies regarding the development of in vitro cortical neurons of rat embryos, $[12,13]$, where it was found that cortical assemblies change their electrophysiological patterns as a function of the network maturation, perhaps because 1000-fold higher synapse density.

Although we observed that both $\mathrm{KCl}$ and 5-HT stimulations were sufficient to trigger firing, we found that only 5-HT efficiently induced a sustained long-lasting activity after the first application.

Serotonin has been frequently associated with changes in excitability. Brief 5-HT application has often been observed to induce immediate hyperexcitability of Aplysia sensory neurons, both in ganglion preparations [31-34] and in cell cultures [35,36]. Moreover, an increase in firing activity elicited by depolarizing current pulses has also been reported in rat cortical neurons after 5-HT exposure [37,38], suggesting a phylogenetically-conserved modulatory action for this neurotransmitter. Therefore, we evaluated the possibility that the observed long-term maintenance of firing activity could result from an increase of cell excitability. In addition to the B2 neuron response, a rapid increase of $\mathrm{C} 1$ neuron excitability was observed without alterations of other parameters, including membrane potential, input resistance and AP threshold. A similar effect was described in the Aplysia metacerebral cell [20,39], homologous to Helix C1 neuron. Even if the modulation of cell excitability may be a good explanation for triggering spontaneous activity in Helix circuits, it cannot be the only effector in promoting its maintenance. Behind the alteration of single cell properties, the emerging evidence would suggest changes in neuronal circuitry features as another putative cause, including the morphological and functional connectivity. 
From a morphological point of view, our results showed an increase of neuritic density in cultures treated with $\mathrm{KCl}$, while a reduction (compared to the nonstimulated networks) occurred in 5-HT stimulated networks. There is a large body of evidence that attribute to 5 -HT an inhibitory role in the regulation of neurite outgrowth in invertebrate models [22-24], thus affecting both the development of arborizations and connectivity formation. In mammals, 5-HT has been implicated in shaping neuronal connectivity, e.g. decreasing neurite branching in rat cortical neurons during development [40] and impairing neurite density in mouse organotypic slice cultures [41].

From a functional point of view, Helix neurons may form both chemical and electrical connections in vitro $[5,7]$. Cross-correlation and latency analysis revealed a strong association between treatment and the type of circuitry connectivity. From the first hours of development, networks treated with 5-HT display synaptic latencies of about $20 \mathrm{~ms}$, compared to the ones treated with $\mathrm{KCl}$ (about $5 \mathrm{~ms}$ after 24 hours). Comparing these values with those measured from interconnected pairs of neurons, we found a striking correspondence between MEA signal latency and the synaptic delays intracellularly recorded (Figure 9F).

The selective presence of the two types of synaptic connections may reflect the different strength of functional links estimated by means of cross-correlation analysis. The occurrence and the magnitude of chemical signal transduction vary dramatically depending on culture conditions, mainly related to the presence of trophic factors [5]. Moreover, phenomena such as neurotransmitter release modulation, synaptic delay, receptor desensitization, signal propagation along neurites and the postsynaptic refractory period may negatively affect the threshold potential to trigger an action potential in the postsynaptic cell. Conversely, the presence of gap junctions facilitate synchronous activity of coupled cells, allowing the direct transmission of electrical signal, thus explaining the 10 -fold higher value of crosscorrelogram peaks measured from $\mathrm{KCl}$-treated cultures.

In support to our results, 5-HT has also been demonstrated to selectively prevent the formation of electrical synapses while allowing chemical synaptogenesis between identified Helisoma neurons [22,42]. The formation of electrical coupling between Helisoma neuronal pairs is thought to require an active growth state and neurite elongation [43-45], negatively modulated by $5-\mathrm{HT}$. However, a direct action of 5-HT on gap junctions has already been demonstrated in Helisoma neurons [46-48], as well as in developing rat cortical neurons [49].

On the other hand, the $\mathrm{KCl}$-induced increase in neurite density may contribute to enhance cell-cell contact, thus promoting a higher coupling coefficient among cells [43]. Since gap junctions likely play a fundamental role in determining network synchronization [50,51], signals may reverberate among neurons until they return to a silent state by switching off the circuit.

Taken together, these observations suggest that the prevalence of chemical connections in 5-HT-treated circuits may be the cause of long-term maintenance of spontaneous activity. In this view, activity may be rapidly triggered by 5 -HT application, through the depolarization of B2 neurons and the increased excitability of C1 neurons, and maintained by the continuous release of neurotransmitter from different firing neurons. Accordingly, repetitive applications of serotonin have previously been implicated in the enhancement of neurotransmitter release through presynaptic mechanisms [52-54].

\section{Conclusions}

In this work, we characterized the dynamics exhibited by networks of Helix neurons coupled to MEAs. By exploiting the technology offered by these devices, we were able to study the development of the network and thus to appreciate possible changes in the network dynamics and firing patterns. In addition, we inferred the functional connections established among the neurons of the networks. Since spontaneously such invertebrate neurons are silent, we triggered their electrophysiological activity by delivering $\mathrm{KCl}$ or $5-\mathrm{HT}$. Under these treatments, we found that: i) $\mathrm{KCl}$ depolarizes the network for brief periods (minutes), without long-term effects. The dynamics of the network is fast $\left(\mathrm{ISI}_{\text {peak }}<0.4\right.$ $\mathrm{ms}$ both for $\mathrm{C} 1$ and B2 cells during the entire development), and the detected functional connections show latencies compatible with electrical synapses $(5.3 \pm 0.3 \mathrm{~ms}$ average value after 48 hours). ii) 5-HT induces longlasting firing activity that persists after the wash-out especially in $\mathrm{B} 2$ neurons. Compared to $\mathrm{KCl}$-induced dynamics, we observed that 5-HT treated networks showed a slower dynamic $\left(\mathrm{ISI}_{\text {peak }}>0.7 \mathrm{~ms}\right.$ both for C1 and B2 cells during the entire development) and signal latencies characterized by delays of $24.7 \pm 0.8 \mathrm{~ms}$, suggesting that these circuits are mediated by chemical synapses. Finally, although the number of functional connections growth as far as the networks develop following a trend comparable with the neuritic outgrowth, we did not find statistically differences in the firing patterns during development as only little deviations-modulations are observed on a rather stable baseline activity.

\section{Methods}

\section{Cell culture}

Juvenile Helix aspersa land snails were purchased from local breeders. Cell cultures were performed as previously described [4]. Briefly, $\mathrm{C} 1$ and B2 neurons were isolated from cerebral and buccal ganglia, respectively, and grown 
under non-adhesive conditions overnight [5]. The day after, Helix somata were plated on poly-L-lysine (Sigma, Milano, Italy) pre-treated MEA devices or plastic dishes [10].

Four polysynaptic circuits (clusters) per MEA with a wellknown composition (two clusters with $2 \mathrm{C} 1+2 \mathrm{~B} 2$, and 2 clusters with $4 \mathrm{~B} 2$, Figure 1A) were assembled taking into account the MEA electrode disposition. Each cell was plated on a single electrode to minimize signal dispersion. We defined as "intra-cluster connectivity" the functional links among the closest cells (i.e., with a Euclidean distance not greater than $d \cdot \sqrt{ } 2$, where $d=200 \mu \mathrm{m}$ is the distance between two electrodes), while as "inter-cluster connectivity" the functional connections among far electrodes (Figure 1A).

The same MEA device was recorded at 6-, 24-, 30-, 48-, 54- and 72 hours after plating.

To investigate the effect of $5-\mathrm{HT}$ and high- $\mathrm{KCl}$ on synaptically-isolated cells, C1 and B2 somata were singularly plated, avoiding cell-to-cell contact, on plastic Petri dishes (Falcon \#1006) coated with poly-L-lysine as adhesive substrate.

\section{Micro-Electrode Arrays, experimental setup and extracellular recordings}

Helix neurons were plated over arrays (MEA 1060, Multi Channel Systems, Reutlingen, Germany) of 60 planar ITO (Indium Tin Oxide) microelectrodes $(30 \mu \mathrm{m}$ diameter, 200 $\mu \mathrm{m}$ spaced) and kept alive in healthy conditions up to 3 days. The experimental set-up is based on the MEA 60 system (Multi Channel Systems, MCS, Reutlingen, Germany): it includes a mounting support with integrated 60 channels, pre- and filter amplifier (gain 1200x), and a personal computer equipped with a PCI data acquisition board for real time signal monitoring and recording. The commercial MC_Rack software (MCS) was used for online visualization and raw data storage; data were further processed by using specifically developed software tools (cf., Data Analysis). Raw data were band pass filtered at 10 $\mathrm{Hz}$ to $3 \mathrm{kHz}$ and sampled at $10 \mathrm{kHz}$ per channel. Figure 1B and $\mathrm{C}$ display 1 second of electrophysiological activity coming from a $\mathrm{C} 1$ (Figure 1B) and a B2 (Figure 1C) neuron.

\section{Intracellular recordings}

Electrophysiological parameters (e.g., membrane potential, input resistance, firing rate and action potential kinetics) were measured using conventional intracellular recordings.

To assess the presence of functional connections in Helix circuitry, intracellular techniques were coupled with MEA recordings in order to selectively stimulate one neuron with a supra-threshold current pulse and observe the propagation of signal among the other interconnected neurons.
To measure synaptic delays in Helix neurons chemically or electrically interconnected, C1-B2 or B2-B2 were paired in culture and electrophysiologically tested 48 hours after plating, as previously described [5]. After that the presence of an electrical or a chemical synapse was determined, cells were recorded during active firing (if necessary, triggered by a short depolarization pulse). Signal traces were then transformed into the first derivate by using GraphPad Prism version 5 (GraphPad Software, San Diego, CA) and the distance between the two related peaks were measured. In chemical synapses, the efficacy of EPSP to trigger an AP is extremely variable, so we defined as "efficacious EPSP" as depolarization event sufficient to reach the threshold potential and elicit a spike into the postsynaptic cell. We decided to include only this category of EPSPs in this analysis, since "notefficacious EPSP" cannot be detected on MEA devices.

\section{Experimental protocol and dataset}

All recordings started $10 \mathrm{~min}$ after positioning the MEAs upon the plate of the amplifier to let the cultures recover from the mechanical and thermal stress due to transfer from the incubator. Recordings were performed at room temperature.

\section{Spontaneous activity - control networks}

Spontaneous activity was recorded for $30 \mathrm{~min}$ after a 10 min recovery period. We repeated such recording at the following time-points: $6,24,30,48,54,72$ hours after the plating. This protocol was applied to three networks composed of $4 \mathrm{C} 1$ and $12 \mathrm{~B} 2$ neurons. The placement of the neurons over the MEA is illustrated in Figure 1A.

\section{Stimulation protocol}

The activity of 5 cultures was recorded at the same time-points considered for the control networks. The complete stimulation protocol consisted of the following steps:

(1) Spontaneous activity was recorded for 30 min after a 10 min recovery period;

(2) Cultures were then stimulated by perfusion of $5-\mathrm{HT}$ or $\mathrm{KCl}$. In particular, 3 cultures were stimulated with 5 - HT, and 2 with $\mathrm{KCl}$. These networks present the same topological placement of the controls (Figure 1A). 5-HT was dissolved in L-15 Leibovitz culture medium to a final concentration of $20 \mu \mathrm{M}$; high potassium physiological salt solution was specifically modified to obtain final concentrations appropriate for Helix $(20 \mathrm{mM} \mathrm{NaCl}, 65 \mathrm{mM} \mathrm{KCl}, 5$ $\mathrm{mM} \mathrm{MgCl} 2,7 \mathrm{mM} \mathrm{CaCl} 2,20 \mathrm{mM}$ HEPES, $\mathrm{pH}$ 7.6; modified from [11]). After 10 minutes cells were rinsed with culture medium $(7.5 \mathrm{ml} / \mathrm{min})$ with 5 volumes of the recording chamber (1 volume $=2 \mathrm{ml})$. 
Flow was actively controlled by a peristaltic pump (Ismatec ISM829; Glattbrugg, Switzerland).

(3) Spontaneous activity was recorded for further 30 min after the stimulation.

\section{Data analysis}

The characterization of the network dynamics exhibited by Helix cultures has been performed by computing the common first-order statistical algorithm, namely mean firing rate (MFR) and inter spike interval (ISI). Functional connectivity was inferred by the computation of the cross-correlation function among the recording sites. A brief description is furnished as follows:

\section{Spike detection and Instantaneous Firing Rate (IFR)}

Spiking activity (Figure 1B and C) exhibited by Helix neurons has been detected by using a previously developed spike detection algorithm named 'Precise Timing Spike Detection' (PTSD) [55]. Briefly, such algorithm makes use of an independent threshold for each channel computed according to the standard deviation of the biological and thermal noise. For the presented experiments we set the spike detection parameters as follows: differential threshold (DT), 8 times the standard deviation of the noise; peak lifetime period $(\mathrm{PLP})=80 \mathrm{~ms}$, refractory period $(\mathrm{RP})=$ $100 \mathrm{~ms}$. The peak lifetime and the refractory period are related to the duration of a spike and the minimum interval between two consecutive events.

The instantaneous firing rate (IFR) is computed by dividing the detected spikes in a small window of size $\Delta t$ by the bin width [56]. Such small window is realized by means of a Gaussian kernel of width equals to $\Delta t=5 \mathrm{~s}$. By dividing the total number of spikes for the entire recording duration, we can estimate the mean firing rate (MFR) of the network.

\section{Inter spike interval (ISI)}

The probability density of time intervals between adjacent spikes is called the inter spike interval (ISI) distribution [57]. Such distribution has been computed by binning the spike train with a time window equal to $20 \mathrm{~ms}$.

\section{Cross-Correlation and functional connectivity maps}

Cross-Correlation (CC) function was built by considering the spike trains of two recording site [19]. The frequency at which a spike firing was recorded in one recording site ('target site') relative to the spike firing in another recording site ('reference site') as a function of time was measured and a CC function was evaluated considering all the pairs of spike trains. Mathematically, $\mathrm{CC}$ reduces to a simple probability $C_{x y}(\tau)$ of observing a spike in a train $Y$ at time $(t+\tau)$, because of a spike in another train $X$ at time $t$; $\tau$ is called time shift or time lag; in this work it was set to $3 \mathrm{~ms}$. Connection strength between the recording sites was evaluated on the basis of the peak value of the CC function, named $C_{\text {peak }}$. Connection directionality was accounted from the sign of the corresponding peak latency. From the statistically relevant $C_{\text {peak }}$, functional connectivity maps were estimated.

Functional connectivity (FC) captures patterns of deviations from statistical independence between distributed and often spatially remote neuronal units [58], measuring their correlation/covariance.

To estimate reliable FC map it is fundamental to threshold the connectivity matrix generated by the CC algorithm in order to consider only "true" and then strongest connections. A procedure to select the strongest functional links is necessary because a $\mathrm{CC}$ value (i.e., a $C_{\text {peak }}$ ) is computed for each electrode pair independently of the existence of a direct or indirect (causal) link, a simply random co-activation or a noisy link. In this analysis, we estimate the FC maps by considering only the strongest links to avoid possible false positive connections and to focus on the most reliable connections (i.e., by considering a small number of links with respect to the total connections identified by the statistical method). In particular, we considered the 8 strongest [26].

\section{Morphological analysis}

At each time-point, MEAs were observed on an Eclipse TE200 inverted microscope (Nikon Instruments, Tokyo, Japan) and images were acquired with a Monochrome Evolution QE camera (MediaCybernetics, Bethesda, MD). Two parameters of neurite outgrowth were assessed: neurite density and velocity of elongation.

With this aim, we considered boxes with fixed area equally distant between two neurons or two clusters of neurons, defined as intra-cluster or inter-cluster type, respectively. For each box, the total number of neurites virtually crossing the selected region were counted and values were divided by the surface area. We refer to this measurement as neurite density.

In order to quantify neurite elongation rates, we trace out 40 neurites from photographs taken sequentially for each MEA and neurite outgrowth was quantitatively measured using a semi-automated analysis system with Image Pro Plus version 6.3 (MediaCybernetics, Bethesda, MD). Neurites were selected for the presence of a growth cone actively growing. Growth velocity rates for each time point were calculated measuring the neurite extension as a function of time and expressed as the percentage of control values.

\section{Statistics}

Data were expressed as means \pm s.e.m. Statistical analysis was performed using GraphPad Prism version 5 (GraphPad Software, San Diego, CA). Statistical significance between group means was assessed using ANOVA analysis (one or two-way and with or without repeated 
measures where appropriate), followed by the Bonferroni's post-hoc test, or by the non-parametric Kruskal-Wallis test, if the normality assumption was not verified (KolmogorovSmirnov normality test). Significance levels were set at $P<0.05$.

\section{Additional file}

Additional file 1: Quasi-periodic behavior of C1 and B2 neurons when treated with 5-HT. (A) Coefficient of variation (CV) of the ISI for C1 (red) and B2 (blue) neurons during development. The dotted gray line shows the threshold (set at 0.2 ) to individuate a quasi-periodic firing activity. (B) Firing activity of the $\mathrm{C} 1$ (red) and B2 (blue) neurons during development. The yellow ellipses mark those frequency values correspondent to a real periodic regime.

\section{Abbreviations}

MEA: Micro-Electrode Array; PTSD: Precise timing spike detection; IFR: Instantaneous firing rate; MFR: Mean firing rate; ISI: Inter spike interval; CC: Cross-correlation; FC: Functional connectivity; AP: Action potential; 5-HT: Serotonin.

\section{Authors' contributions}

PM and CNGG, designed and performed the experiments; PM and CNGG analyzed the data; MG, SM helped interpret the results, and contributed to the preparation of the manuscript. All the authors participated to the manuscript writing. All authors read and approved the final manuscript.

\section{Acknowledgments}

The authors are very grateful to Prof. Pier Giorgio Montarolo for the thorough revision of the manuscript and his valuable suggestions. This work is partially supported by Compagnia di San Paolo (grant n: 2008.2363).

\section{Author details}

'Neuroengineering and Bio-nano Technology Group (NBT), Department of Informatics, Bioengineering, Robotics, System Engineering (DIBRIS), University of Genova, Genova, Italy. ${ }^{2}$ Department of Neuroscience, University of Torino, Torino, Italy. ${ }^{3}$ stituto Nazionale di Neuroscienze, Torino, Italy.

Received: 3 October 2012 Accepted: 18 February 2013

Published: 25 February 2013

\section{References}

1. Altrup U, Speckmann EJ: Identified neuronal individuals in the buccal ganglia of Helix pomatia. Neurosci Behav Physiol 1994, 24:23-32.

2. Kononenko Nl: Excitatory and inhibitory monosyanptic peptidercic transmission in the CNS of the snail Helix pomatia. Neurosci Behav Physiol 1994, 24:203-208.

3. Bulloch AGM, Syed NI: Reconstruction of neuronal networks in culture. Trends Neurosci 1992, 15:422-427.

4. Ghirardi M, Casadio A, Santarelli L, Montarolo PG: Aplysia hemolymph promotes neurite outgrowth and synaptogenesis of identified Helix neurons in cell culture. Invert Neurosci 1996, 2:41-49.

5. Fiumara F, Leitinger G, Milanese C, Montarolo PG, Ghirardi M: In vitro formation and activity-dependent plasticity of synapses between helix neurons involved in the neural control of feeding and withdrawal behaviors. Neuroscience 2005, 134:1133-1151.

6. Fiumara F, Milanese C, Corradi A, Giovedi S, Leitinger G, Menegon A, Montarolo PG, Benfenati F, Ghirardi M: Phosphorylation of synapsin domain A is required for post-tetanic potentiation. J Cell Sci 2007, 120:3228-3237.

7. Giachello CN, Fiumara F, Giacomini C, Corradi A, Milanese C, Ghirardi M, Benfenati F, Montarolo PG: MAPK/Erk-dependent phosphorylation of synapsin mediates formation of functional synapses and short-term homosynaptic plasticity. J Cell Sci 2010, 123:881-893.

8. Giachello CN, Montarolo PG, Ghirardi M: Synaptic functions of invertebrate varicosities: what molecular mechanisms lie beneath. Neural Plast 2012, 2012:670821.
9. Kleinfeld D, Raccuia-Behling F, Chiel HJ: Circuits constructed from identified Aplysia neurons exhibit multiple patterns of persistent activity. Biophys J 1990, 57:697-715.

10. Massobrio P, Tedesco M, Giachello C, Ghirardi M, Fiumara F, Martinoia S: Helix neuronal ensembles with controlled cell type composition and placement develop functional polysynaptic circuits on Micro-Electrode Arrays. Neurosci Lett 2009, 467:121-126.

11. Cottrell GA, Macon JB: Synaptic connexions of two symmetrically placed giant serotonin-containing neurones. J Physiol 1974, 236:435-464.

12. Chiappalone M, Bove M, Vato A, Tedesco M, Martinoia S: Dissociated cortical networks show spontaneously correlated activity patterns during in vitro development. Brain Res 2006, 1093:41-53.

13. Wagenaar DA, Pine J, Potter SM: An extremely rich repertoire of bursting patterns during the development of cortical cultures. BMC Neurosci 2006, 7:11.

14. Van Pelt J, Vajda I, Wolters PS: Corner MA, Ramakers GJA: Dynamics and plasticity in developing neural networks in vitro. Prog Brain Res 2005, 147:173-188.

15. Van Pelt J, Wolters PS, Corner MA, Rutten WLC, Ramakers GJA: Long-term characterization of firing dynamics of spontaneous bursts in cultured neural networks. IEEE Trans Biomed Eng 2004, 51:2051-2062.

16. Downes JH, Hammond MW, Xydas D, Spencer MC, Becerra VM, Warwick K, Whalley BJ, Nasuto SJ: Emergence of a Small-World Functional Network in Cultured Neurons. PLoS Comput Biol 2012, 8:e1002522.

17. Pasquale V, Massobrio P, Bologna LL, Chiappalone M, Martinoia S: Selforganization and neuronal avalanches in networks of dissociated cortical neurons. Neuroscience 2008, 153:1354-1369.

18. Claverol-Tinturé E, Rossel X, Cabestany J: Technical steps towards one-to -one electrode-neuron interfacing with neural circuits reconstructed in vitro. Neurocomputing 2007, 70:2716-2722.

19. Garofalo M, Nieus T, Massobrio P, Martinoia S: Evaluation of the performance of information theory-based methods and cross-correlation to estimate the functional connectivity in cortical networks. PLoS One 2009, 4:e6482.

20. Jacklet JW, Grizzaffi J, Tieman DG: Serotonin and cAMP induce excitatory modulation of a serotonergic neuron. J Neurobiol 2006, 66:499-510.

21. Weiss KR, Chiel HJ, Koch U, Kupfermann I: Activity of an identified histaminergic neuron, and its possible role in arousal of feeding behavior in semi-intact Aplysia. J Neurosci 1986, 6:2403-2415.

22. Haydon PG, McCobb DP, Kater SB: Serotonin selectively inhibits growth cone motility and synaptogenesis of specific identified neurons. Science 1984, 226:561-564.

23. Haydon PG, McCobb DP, Kater SB: The regulation of neurite outgrowth, growth cone motility, and electrical synaptogenesis by serotonin. J Neurobiol 1987, 18:197-215.

24. Goldberg $\mathrm{J}$ : Serotonin regulation of neurite outgrowth in identified neurons from mature and embryonic Helisoma trivolvis. Perspect Dev Neurobiol 1998, 5:373-387.

25. Kanagasabapathi TT, Massobrio P, Barone RA, Tedesco M, Martinoia S, Wadman WJ, Decre MMJ: Functional connectivity and dynamics of cortical-thalamic networks co-cultured in a dual compartment device. J Neural Eng 2012, 9(3):036010.

26. Maccione A, Garofalo M, Nieus T, Tedesco M, Berdondini L, Martinoia S: Multiscale functional connectivity estimation on low-density neuronal cultures recorded by high-density CMOS Micro Electrode Arrays. J Neurosci Methods 2012, 207:161-171.

27. Martinoia S, Massobrio P, Bove M, Massobrio G: Cultured neurons coupled to microelectrode arrays: circuit models, simulations and experimental data. IEEE Trans Biomed Eng 2004, 51:859-864.

28. Massobrio P, Massobrio G, Martinoia S: Multi-program approach for simulating recorded extracellular signals generated by neurons coupled to microelectrode arrays. Neurocomputing 2007, 70:2467-2476.

29. Fromherz $\mathrm{P}$, In Nanoelectronics and Information Technology: Neuroelectronic interfacing: semiconductor chips with ion channels, nerve cells, and brain. Berlin: Wiley-VCH: Edited by Waser R; 2003:781-810.

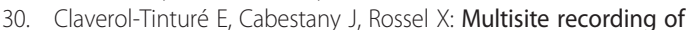
extracellular potentials produced by microchannel-confined neurons invitro. IEEE Trans Biomed Eng 2007, 54:331-335.

31. Baxter DA, Byrne JH: Differential effects of cAMP and serotonin on membrane current, action-potential duration, and excitability in somata of pleural sensory neurons of Aplysia. J Neurophysiol 1990, 64:978-990. 
32. Klein M, Hochner $B$, Kandel ER: Facilitatory transmitters and CAMP can modulate accommodation as well as transmitter release in Aplysia sensory neurons: Evidence for parallel processing in a single cell. Proc Natl Acad Sci USA 1986, 83:7994-7998.

33. Stark LL, Mercer AR, Emptage NJ, Carew TJ: Pharmacological and kinetic characterization of two functional classes of serotonergic modulation in Aplysia sensory neurons. J Neurophysiol 1996, 75:855-866.

34. Walters ET, Byrne JH, Carew TJ, Kandel ER: Mechanoafferent neurons innervating tail of Aplysia. II. Modulation by sensitizing stimulation. J Neurophysiol 1983, 50:1543-1559.

35. Dale N, Kandel ER, Schacher S: Serotonin produces long-term changes in the excitability of Aplysia sensory neurons in culture that depend on new protein synthesis. J Neurosci 1987, 7:2232-2238.

36. Ghirardi M, Braha O, Hochner B, Montarolo PG, Kandel ER, Dale N: Roles of PKA and PKC in facilitation of evoked and spontaneous transmitter release at depressed and nondepressed synapses in Aplysia sensory neurons. Neuron 1992, 9:479-489.

37. Araneda R, Andrade R: 5-Hydroxytryptamine2 and 5-hydroxytryptamine $1 \mathrm{~A}$ receptors mediate opposing responses on membrane excitability in rat association cortex. Neuroscience 1991, 40:399-412.

38. Zhong P, Yuen EY, Yan Z: Modulation of neuronal excitability by serotonin-NMDA interactions in prefrontal cortex. Mol Cell Neurosci 2008 , 38:290-299.

39. Weiss KR, Shapiro E, Kupfermann I: Modulatory synaptic actions of an identified histaminergic neuron on the serotonergic metacerebral cell of Aplysia. J Neurosci 1986, 6:2393-2402.

40. Sikich L, Hickok JM, Todd RD: 5-HT1A receptors control neurite branching during development. Dev Brain Res 1990, 56:269-274.

41. Dudok JJ, Groffen AJ, Witter MP, Voorn P, Verhage M: Chronic activation of the 5-HT(2) receptor reduces 5-HT neurite density as studied in organotypic slice cultures. Brain Res 2009, 1302:1-9.

42. Haydon PG, Kater SB: The differential regulation of formation of chemical and electrical connections in Helisoma. J Neurobiol 1988, 19:636-655.

43. Hadley RD, Bodnar DA, Kater SB: Formation of electrical synapses between isolated, cultured Helisoma neurons requires mutual neurite elongation. J Neurosci 1985, 5:3145-3153.

44. Hadley RD, Kater SB: Competence to form electrical connections is restricted to growing neurites in the snail, Helisoma. J Neurosci 1983, 3:924-932.

45. Hadley RD, Kater SB, Cohan CS: Electrical synapse formation depends on interaction of mutually growing neurites. Science 1983, 221:466-468.

46. Berdan RC, Shivers RR, Bulloch AG: Chemical synapses, particle arrays, pseudo-gap junctions and gap junctions of neurons and glia in the buccal ganglion of Helisoma. Synapse 1987, 1:304-323.

47. Guthrie PB, Lee RE, Rehder V, Schmidt MF, Kater SB: Self-recognition: a constraint on the formation of electrical coupling in neurons. J Neurosci 1994, 14:1477-1485.

48. Szabo TM, Caplan JS, Zoran MJ: Serotonin regulates electrical coupling via modulation of extrajunctional conductance: H-current. Brain Res 2010, 1349:21-31

49. Rorig B, Sutor B: Serotonin regulates gap junction coupling in the developing rat somatosensory cortex. Eur J Neurosci 1996, 8:1685-1695.

50. Bennett MV, Zukin RS: Electrical coupling and neuronal synchronization in the mammalian brain. Neuron 2004, 41:495-511.

51. Kavasseri RG, Nagarajan R: Synchronization in electrically coupled neura networks. Complex Syst 2006, 16:369-380.

52. Klein M, Kandel ER: Mechanism of calcium current modulation underlying presynaptic facilitation and behavioral sensitization in Aplysia. Proc Natl Acad Sci USA 1980, 77:6912-6916.

53. Delaney K, Tank DW, Zucker RS: Presynaptic calcium and serotoninmediated enhancement of transmitter release at crayfish neuromuscular junction. J Neurosci 1991, 11:2631-2643.

54. Katz PS, Frost WN: Intrinsic neuromodulation in the Tritonia swim CPG: the serotonergic dorsal swim interneurons act presynaptically to enhance transmitter release from interneuron C2. J Neurosci 1995, 15:6035-6045.
55. Maccione A, Gandolfo M, Massobrio P, Novellino A, Martinoia S, Chiappalone M: A novel algorithm for precise identification of spikes in extracellularly recorded neuronal signals. J Neurosci Methods 2009, 177:241-249.

56. Rieke F, Warland D, de Ruyter van Steveninck R, Bialek W: Spikes: exploring the neural code. Cambridge, Massachusetts: The MIT Press; 1997.

57. Dayan P, Abbott LF: Theoretical neuroscience. Cambridge: MIT; 2001.

58. Friston KJ: Functional and effective connectivity in neuroimaging: a syntesis. Hum Brain Mapp 1994, 2:56-78.

doi:10.1186/1471-2202-14-22

Cite this article as: Massobrio et al:: Selective modulation of chemical and electrical synapses of Helix neuronal networks during in vitro development. BMC Neuroscience 2013 14:22.

\section{Submit your next manuscript to BioMed Central and take full advantage of:}

- Convenient online submission

- Thorough peer review

- No space constraints or color figure charges

- Immediate publication on acceptance

- Inclusion in PubMed, CAS, Scopus and Google Scholar

- Research which is freely available for redistribution

Submit your manuscript at www.biomedcentral.com/submit
C) BioMed Central 